\title{
Serum levels of high-sensitivity troponin T: a novel marker for left ventricular remodeling and performance in hypertensive subjects
}

\author{
D.M. Miao*, L.P. Zhang*, H.P. Yu*, J.Y. Zhang, W.K. Xiao and P. Ye \\ Department 2 of Geriatric Cardiology, Chinese PLA General Hospital, \\ Beijing, China \\ *These authors contribued equally to this study. \\ Corresponding author: P. Ye \\ E-mail: PingYEcn@163.com
}

Genet. Mol. Res. 13 (3): 5143-5153 (2014)

Received June 11, 2013

Accepted October 4, 2013

Published July 7, 2014

DOI http://dx.doi.org/10.4238/2014.July.7.7

\begin{abstract}
It has been well established that high-sensitivity cardiac troponin $\mathrm{T}$ (hs-TnT) is a specific and highly sensitive marker in acute coronary syndromes. On the other hand, studies on serum concentrations of hs-TnT in patients with hypertension in the absence of significant coronary stenosis are limited. Therefore, we hypothesized that hs-TnT levels are related to left ventricular (LV) remodeling and performance in hypertension. We included 537 hemodynamically stable hypertensive subjects, 247 males aged $60.7 \pm 11.1$ years, and 100 normotensive subjects of similar age and gender. Clinical examination, clinical assessment and laboratory assays were performed for all hypertensive and normotensive subjects. The detectable rate $(>0.003 \mathrm{ng} / \mathrm{mL})$ and elevated rate $(>0.013 \mathrm{ng} / \mathrm{mL})$ of hs-TnT were higher in hypertensive subjects than those in normotensive subjects. hs-TnT level gradually increased in hypertensive subjects with LV normal geometry, concentric remodeling, concentric hypertrophy and eccentric hypertrophy. hs-TnT was independently related to age, gender, hypertension, fasting blood glucose, renal function, and LV
\end{abstract}


hypertrophy, and diastolic function on multiple analysis during the whole participation. An increase in hs-TnT levels could be a reliable biomarker of cardiac remodeling and function in hypertension, as an indicator of subclinical ongoing cardiomyocyte injury.

Key words: High-sensitivity troponin T; Hypertension; Cardiac remodeling; Diastolic function

\section{INTRODUCTION}

Cardiac troponin is a kind of regulatory proteins that controls the calcium-mediated interaction of actin and myosin (Antman, 2002). Due to its sensitivity and specificity for detection of cardiomyocyte injury, it is well known that cardiac troponin levels predict myocardial ischemia (Thygesen et al., 2007).

Recent advances have led to the development of highly sensitive troponin assays that possess greater sensitivity than previous assays and improved precision at lower limits of detection (Apple et al., 2007). Several studies have reported that highly sensitive serum cardiac troponin $\mathrm{T}$ (hs-TnT) is a specific and high-sensitivity marker of myocardial injury (Apple et al., 2007; Reichlin et al., 2009). It not only provides good sensitivity and specificity for acute coronary syndrome but also for stable angina (Omland et al., 2009; Reichlin et al., 2009). In addition, elevated levels of hs-TnT have been associated with metabolic syndrome (Siervo et al., 2010), and hs-TnT is the most powerful predictor of cardiovascular disease in patients with dialysis-dependent chronic renal failure. (McGill et al., 2010). Recently, it was reported that hs-TnT correlated with the severity and prognosis of hypertrophic cardiomyopathy, which may be the result of myocyte apoptosis and increased interstitial fibrosis (Sato et al., 2003; Moreno et al., 2010).

Our previous study about the association between hs-TnT and predicted cardiovascular risks in a community-based population showed that multiple conventional risk factors such as hypertension were associated with higher hs-TnT concentrations (Xu et al., 2011). Increased myocardial strain due to pressure or volume overload may be important causes of cardiac injury (Ganau et al., 1992). For these same reasons, hypertension is often associated with myocardial hypertrophy, myocyte damage and interstitial fibrosis, which contribute to hypertensive cardiac remodeling and abnormal cardiac performance (Koh et al., 2009).

Studies of serum concentrations of hs-TnT in subjects with hypertension without overt cardiovascular diseases are limited. Therefore, we hypothesized that an increase in hsTnT levels would be related to left ventricular (LV) remodeling and function in hypertension. In this setting, we tried to explore the association between hs-TnT and clinical variables and LV remodeling and function in hypertensive subjects.

\section{MATERIAL AND METHODS}

\section{Study population}

We enrolled hypertensive subjects in clinical outpatients in PLA General Hospital in China, from January 2012 to January 2013. Subjects with mean values of three mea- 
surements of systolic arterial blood pressure $\geq 140 \mathrm{mmHg}$ and/or diastolic arterial blood pressure $\geq 90 \mathrm{mmHg}$ at rest, and/or use of antihypertensive medication, were defined as having hypertension. Exclusion criteria included the presence of overt cardiovascular disease, renal dysfunction, bedridden status, mental illness, severe systemic disease, acute and chronic inflammatory diseases, malignancy and any condition preventing technically adequate echocardiography evaluation. Eventually, 537 hypertensive subjects were eligible for the study. These subjects were in a stable hemodynamic condition without obvious discomfort needed for hospitalization. At the same time, 100 normotensive subjects of similar age and gender were enrolled as the control group. Subjects gave signed consent that permitted the use of collected data for research without disclosure of personal identity. The study protocol was approved by the local Research Ethics Committee of PLA General Hospital Beijing, China.

\section{Definition of variables}

Cardiovascular disease was defined as self-reported history of angina, myocardial infarction, previously documented angiographic disease, positive stress test, coronary or peripheral arterial revascularization, heart failure, cerebrovascular disease and stroke. Subjects with fasting glucose $\geq 6.99 \mathrm{mM}$, 2-h plasma glucose $\geq 11.1 \mathrm{mM}$, or taking insulin injection or anti-diabetic agents were defined as having diabetes mellitus. Renal dysfunction was defined as an estimated glomerular filtration rate (eGFR) $<60 \mathrm{~mL} / \mathrm{min}$ per $1.73 \mathrm{~m}^{2}$. Characteristics of the study population are listed in Table 1 .

\begin{tabular}{|c|c|c|}
\hline Clinical variable & Hypertensive group $(\mathrm{N}=537)$ & Normotensive group $[\mathrm{N}=100$, subjects $(\mathrm{N}=72)]$ \\
\hline Age (years) & $60.7 \pm 11.1$ & $59.5 \pm 10.7$ \\
\hline Male $(\%)$ & $247(46.0)$ & $45(45.0)$ \\
\hline BMI $\left(\mathrm{kg} / \mathrm{m}^{2}\right)$ & $25.7 \pm 3.30 *$ & $24.3 \pm 2.38$ \\
\hline Smoking (\%) & $155(28.8)^{\wedge}$ & $23(23.0)$ \\
\hline Diabetes $(\%)$ & $158(27.6)^{\wedge}$ & $19(19.0)$ \\
\hline $\mathrm{SBP}(\mathrm{mmHg})$ & $140.1 \pm 12.3^{\wedge}$ & $128.5 \pm 12.6$ \\
\hline $\mathrm{DBP}(\mathrm{mmHg})$ & $80.2 \pm 10.7^{\wedge}$ & $75.4 \pm 9.3$ \\
\hline $\mathrm{FBG}(\mathrm{mM})$ & $5.8 \pm 1.5^{\wedge}$ & $5.3 \pm 0.6$ \\
\hline $\mathrm{TG}(\mathrm{mM})$ & $1.8(1.3-2.2)^{*}$ & $1.7(1.1-2.3)$ \\
\hline $\mathrm{TC}(\mathrm{mM})$ & $5.2 \pm 0.6^{*}$ & $4.8 \pm 0.9$ \\
\hline HDL-c (mM) & $1.0 \pm 0.3^{\wedge}$ & $1.2 \pm 0.3$ \\
\hline LDL-c (mM) & $3.3 \pm 0.6^{\wedge}$ & $3.1 \pm 0.6$ \\
\hline $\operatorname{Egfr}\left(\mathrm{mL} / \mathrm{min} / 1.73 \mathrm{~m}^{2}\right)$ & $87.2 \pm 16.5^{\wedge}$ & $90.0 \pm 15.0$ \\
\hline $\operatorname{TnT}(\mathrm{pg} / \mathrm{mL}) \mathrm{M}$ & $5.8(3.0-12.7)^{\wedge}$ & $3.1(3.0-7.0)$ \\
\hline \multicolumn{3}{|l|}{ Medications } \\
\hline CCB $(\%)$ & $201(37.4)^{\wedge}$ & 0 \\
\hline ACEI/ARB (\%) & $93(17.3)^{\wedge}$ & 0 \\
\hline Diuretic $(\%)$ & $56(10.4)^{\wedge}$ & 0 \\
\hline$\beta$-blocker $(\%)$ & $114(21.2)^{\wedge}$ & 0 \\
\hline
\end{tabular}

The normal distributed continuous variables are reported as means $\pm \mathrm{SD}$, and the nonparametrically distributed are reported as median (interquartile range). Categorical variables were presented as frequencies (percentages). BMI $=$ body mass index; $\mathrm{SBP}=$ systolic blood pressure; $\mathrm{DBP}=$ diastolic blood pressure; $\mathrm{FBG}=$ fasting blood glucose; $2 \mathrm{~h}$ $\mathrm{PBG}=2 \mathrm{~h}$ postprandial blood glucose; $\mathrm{TG}=$ triglycerides; $\mathrm{TC}=$ total plasma cholesterol; HDL-c $=$, high-density lipoprotein cholesterol; LDL-c $=$ low-density lipoprotein cholesterol; eGFR = estimated glomerular filtration rate; hs- $\mathrm{TnT}=$ high-sensitivity troponin $\mathrm{T} ; \mathrm{CCB}=$ Calcium blocker; $\mathrm{ACEI}=$ angiotensin-converting enzyme inhibitor; $\mathrm{ARB}=$ angiotensin II receptor blocker. ${ }^{*} \mathrm{P}<0.05$ hypertension $v$ s normotension. ${ }^{\wedge} \mathrm{P}<0.001$ hypertension vs normotension. 


\section{Transthoracic echocardiography}

Comprehensive examinations including M-mode, two-dimensional conventional Doppler, and color Doppler echocardiography were performed using the $\alpha-10$ apparatus (Aloka, Tokyo, Japan) with a 1.5-2.5 MHz transducer. Penn convention measurements were used to calculate LV mass and it was indexed by body surface area. American Society of Echocardiography measurements (Sahn et al., 1978) were used for LV chamber diameter, wall thickness, and relative wall thickness.

Echocardiographic parameters were measured by the consensus of two experienced investigators who were blinded to the metabolic data of the subjects. Measurements were taken as a mean of five beats. The inter-observer variability of ultrasound measurements for the normotensive subjects is described in Table 2.

\begin{tabular}{|c|c|c|c|}
\hline $\begin{array}{l}\text { Variables } \\
\end{array}$ & First observer & Second observer & ICC \\
\hline IVSTd (mm) & $9.6 \pm 1.9$ & $9.5 \pm 2.0$ & 0.98 \\
\hline LVMI $\left(\mathrm{g} / \mathrm{m}^{2}\right)$ & $94.7 \pm 20.2$ & $97.5 \pm 23.9$ & 0.95 \\
\hline $\operatorname{LAD}(\mathrm{mm})$ & $29.2 \pm 3.6$ & $29.7 \pm 4.8$ & 0.96 \\
\hline LVEDd (mm) & $42.3 \pm 1.44$ & $42.8 \pm 1.37$ & 0.97 \\
\hline $\operatorname{LVEF}(\%)$ & $68.7 \pm 6.8$ & $67.5 \pm 5.3$ & 0.97 \\
\hline mFS (\%) & $25.2 \pm 3.6$ & $25.8 \pm 2.9$ & 0.96 \\
\hline E/E'ratio & $5.8 \pm 1.5$ & $6.4 \pm 1.2$ & 0.95 \\
\hline
\end{tabular}

Tissue Doppler measurements were made in four myocardial segments: longitudinal function from the velocities of basal septal and lateral segments, and basal anterior and basal inferior segments. Peak myocardial velocities in systole and early diastole were measured. Velocities of the four segments imaged were averaged. LV diastolic filling pressure was estimated from E/E' ratio [the ratio of mitral E wave, by pulsed wave Doppler, to mitral annular early diastolic velocity (E'wave) by pulsed wave tissue Doppler]. (Arques et al., 2007).

\section{Definition of variables}

$\mathrm{LV}$ mid-wall fractional shortening $(\mathrm{mFS})=[(\mathrm{LVEDd}+\mathrm{PWTd} / 2+\mathrm{IVSTd} / 2)-($ LVEDs + PWTs/2 + IVSTs/2)] / (LVEDd + PWTd/2 + IVSTd/2) (de Simone and Devereux, 2002). LV mass $($ LVM $)=1.04 \times\left[(\text { LVEDd }+ \text { IVSTd }+ \text { PWTd })^{3}-\right.$ LVEDd $\left.^{3}\right]-13.6$. Relative wall thickness $($ RWT $)=2$ PWTd / LVEDd (Devereux et al., 1986). IVSTd means end-diastolic thickness of LV septum; IVSTs is end-systolic thickness of LV septum; PWTd is end-diastolic thickness of LV postal wall; PWTs is end-systolic thickness of LV postal wall; LVEDd is LV end-diastolic diameter; and LVEDs is LV end-systolic diameter.

\section{Echo classifications}

Classification of patients based on whether LV muscle mass and the LV wall thick- 
ness/chamber radius ratio ("relative wall thickness") are normal or abnormal yields four groups with different LV geometric patterns: normal geometry (normal mass and relative wall thickness), concentric remodeling (increased relative wall thickness with normal mass]), concentric hypertrophy (an increase in LV mass with normal relative wall thickness), and eccentric hypertrophy (increased LV mass with normal wall thickness) (Devereux et al., 1986). Specifically, the normal geometry group showed LVMI $<125 \mathrm{~g} / \mathrm{m}^{2}$ (male) or $<120 \mathrm{~g} / \mathrm{m}^{2}$ (female) and RWT $<0.45$, and the concentric remodeling group showed LVMI $<125 \mathrm{~g} / \mathrm{m}^{2}$ (male) or $<120 \mathrm{~g} / \mathrm{m}^{2}$ (female) and RWT $\geq 0.45$. The concentric hypertrophy group showed LVMI $\geq 125 \mathrm{~g} / \mathrm{m}^{2}$ (male) or $\geq 120 \mathrm{~g} / \mathrm{m}^{2}$ (female) and RWT $\geq 0.45$, and the eccentric hypertrophy group showed LVMI $\geq 125 \mathrm{~g} / \mathrm{m}^{2}$ (male) or $\geq 120 \mathrm{~g} / \mathrm{m}^{2}$ (female) and RWT $<0.45$ (Ganau et al., 1992).

\section{Blood samples and laboratory assays}

Venipuncture was performed in the morning on patients and healthy control subjects after overnight fasting and maintained at $4^{\circ} \mathrm{C}$ for $\leq 2 \mathrm{~h}$ before being centrifuged at $1200 \mathrm{~g}$ for $15 \mathrm{~min}$. Serum aliquots were frozen at $-80^{\circ} \mathrm{C}$ until assays were performed. Concentrations of fasting glucose, total cholesterol, triglycerides, and high-density and low-density lipoprotein cholesterol (HDL-C and LDL-C) were determined using Roche enzymatic assays (Roche Diagnostics GmbH, Mannheim, Germany) on a Roche autoanalyzer (Roche Diagnostics, Indianapolis, IN, USA). Concentrations of hs-TnT were determined using an Elecsys Troponin T high-sensitivity assay (Roche Diagnostics GmbH, Mannheim, Germany) by electrochemiluminescence immunoassay on a Modular Analytics E170 autoanalyzer (Roche Diagnostics). Given the enhanced sensitivity, this assay was reported in $\mathrm{pg} / \mathrm{mL}$ units and it had an analytical measurement range of 3 to $10,000 \mathrm{pg} / \mathrm{mL}$ (according to manufacturer information). Concentrations of serum creatinine were measured by enzymatic assay (Roche Diagnostics GmbH) on a Hitachi 7600 autoanalyzer (Hitachi, Tokyo, Japan). Serum creatinine was standardized using a calibration equation: Jaffe's kinetic method Scr (mg/dL) $=0.795 \times$ [enzymatic method Scr $(\mathrm{mg} / \mathrm{dL})]+0.29$. The estimated glomerular filtration rate (eGFR) was calculated with the Chinese Modification of Diet in Renal Disease (C-MDRD) equation: eGFR $\left(\mathrm{mL} / \mathrm{min}\right.$ per $\left.1.73 \mathrm{~m}^{2}\right)=175 \mathrm{x}$ standardized creatinine $(\mathrm{mg} / \mathrm{dL})^{-1.234} \mathrm{x}$ age (years) $)^{-0.179} \times 0.79$ (if female). All testing was performed by well-trained personnel blinded to the clinical data in the Department of Biochemistry of Chinese PLA General Hospital.

\section{Statistical analysis}

The normal distributed continuous variables are reported as means $\pm \mathrm{SD}$, and the non parametrically distributed ones are reported as medians (interquartile range). Categorical variables were presented as frequencies (percentages). hs-TnT was classified as undetectable $(<3.0 \mathrm{pg} / \mathrm{mL})$, detectable $(\geq 3.0 \mathrm{pg} / \mathrm{mL})$, and elevated $(\geq 13.3 \mathrm{pg} / \mathrm{mL})$ (Wallace et al., 2006). An unpaired $t$-test or Mann-Whitney $\mathrm{U}$ test was used for comparison of continuous variables between two groups. A chi-square test was used for comparison of groups of categorical variables. Consistency check was performed for the inter-observer variability of ultrasound measurements. hs-TnT levels (detectable and elevated) in different cardiac remodeling groups were compared using the multivariable logistic regression procedures to calculate adjusted odds ratios (ORs). Modeled were age, gender, current smoking, body mass index, systolic 
blood pressure, diastolic blood pressure, fasting blood glucose, triglycerides, total plasma cholesterol, HDL-C, LDL-C, estimated glomerular filtration rate, and usage of antihypertenive drugs. Correlation between two continuous variables was performed by the Pearson test. Continuous variables were tested for normal distribution by the Kolmogorov-Smirnov test. Variables were log-transformed before being included in the analysis if they were not normally distributed. Stepwise multiple regression analyses were performed to identify independent predictors of hs-TnT concentrations requiring a probability value of $\leq 0.10$ to be entered and $\leq 0.05$ to remain in the model. Parameters included as potential independent variables were age, gender, smoking ratio, systolic blood pressure, diastolic blood pressure, fasting glucose, eGFR, and echocardiographic parameters, including left atrial diameter, mid-wall fractional shortening, E/E' ratio, and LVMI. All statistical procedures were performed using SPSS statistical package, version 13.0 (SPSS Inc. Chicago, Illinois, USA). P $<0.05$ was considered to be statistically significant.

\section{RESULTS}

\section{Characteristics of the study participants}

The baseline characteristics of participants are shown in Table 1. In the hypertensive subjects, age ranged from 45 to 76 years old, and male percentage was $46.0 \%$. The distribution of age and gender did not differ between groups, while their age, current smoking ratio, body mass index, triglycerides, total plasma cholesterol, HDL-C, LDL-C, blood glucose, and blood pressure were obviously higher than those in the normotensive subjects. Furthermore, eGFR was lower than that in normotensives $(\mathrm{P}<0.001)$. With respect to anti-hypertensive drugs, the usage of calcium blocker, angiotensin-converting enzyme inhibitor/angiotensin receptor blocker, diuretic and $\beta$-blocker was $37.4,17.3,10.4$, and $21.2 \%$, respectively (Table 1 ).

\section{Clinical variables in different hypertensive remodeling}

Subjects in the four groups characterized by different LV geometric patterns were similar regarding gender distribution, body mass index and different usage of antihypertensive drugs $(\mathrm{P}$ $>0.05$ ). Patients with the pattern of LV concentric and eccentric hypertrophy had higher values for age, smoking, diabetes, blood pressures, fasting blood glucose and blood lipids, while eGFR was decreased in the eccentric hypertrophy group compared with the other groups (Table 3).

\section{Distribution of hs-TnT in subjects with various hypertensive remodeling groups}

Of the 537 hypertensive participants without overt CVD, 419 subjects (78.0\%) had detectable hs-TnT values $(>3.0 \mathrm{pg} / \mathrm{mL})$ and 165 subjects $(30.7 \%)$ had elevated hs-TnT $(>13.3 \mathrm{pg} /$ $\mathrm{mL})$. The prevalence of detectable hs-TnT in the normotension, normal geometry, concentric remodeling, concentric hypertrophy, and eccentric hypertrophy groups were $46.0 \%(46 / 100)$, $61.5 \%(112 / 182), 70.1 \%(89 / 127), 94.5 \%(155 / 164)$, and $96.9 \%(62 / 64)$, respectively, while the prevalence of elevated hs-TnT was 8.0\% (8/100), 11.0\% (20/182), 16.5\% (21/127), 49.4 (81/164), and $65.5(42 / 64)$, respectively. The prevalence of detectable hs-TnT in the hypertensive group was significantly higher than that in the normotensive group $(\mathrm{OR}=2.73,95 \% \mathrm{CI}=1.80-3.60, \mathrm{P}$ 
$<0.001)$. The prevalence of elevated hs-TnT in the hypertensive group was significantly higher than that in normotensive group $(\mathrm{OR}=3.21,95 \% \mathrm{CI}=3.08-4.02, \mathrm{P}<0.001)$. The prevalence of detectable and elevated hs-TnT differed significantly between the different hypertensive remodeling groups, except for the concentric remodeling and the normal geometry groups (Table 4).

\begin{tabular}{|c|c|c|c|c|}
\hline Variables No. (\%) & $\begin{array}{c}\text { Normal geometry } \\
182(33.9)\end{array}$ & $\begin{array}{c}\text { Concentric remodeling } \\
127(27.6)\end{array}$ & $\begin{array}{c}\text { Concentric hypertrophy } \\
164(30.5)\end{array}$ & $\begin{array}{c}\text { Eccentric hypertrophy } \\
64(11.9)\end{array}$ \\
\hline Age (years) & $58.1 \pm 8.6$ & $59.6 \pm 9.0$ & $61.0 \pm 8.8^{*}$ & $64.7 \pm 9.5^{* \#}$ \\
\hline Male $(\%)$ & $92(50.5)$ & $63(49.6)$ & $67(40.9)$ & $25(39.1)$ \\
\hline BMI (kg/m2) & $25.3 \pm 2.3$ & $25.6 \pm 2.4$ & $25.7 \pm 2.3$ & $25.9 \pm 3.7$ \\
\hline Smoking (\%) & $35(19.2)$ & $36(28.8)$ & $67(40.9)^{* \wedge}$ & $29(45.0)^{* \wedge}$ \\
\hline Diabetes (\%) & $20(11.0)$ & $20(15.7)$ & $67(40.8)^{* \wedge}$ & $51(79.7)^{* \wedge \#}$ \\
\hline $\mathrm{SBP}(\mathrm{mmHg})$ & $129.4 \pm 8.0$ & $135.0 \pm 9.8^{*}$ & $145.4 \pm 11.3^{* \wedge}$ & $152.1 \pm 9.4^{* \wedge \#}$ \\
\hline $\mathrm{DBP}(\mathrm{mmHg})$ & $75.2 \pm 10.1$ & $77.0 \pm 12.4$ & $82.2 \pm 6.9^{* \wedge}$ & $83.0 \pm 9.2^{* \wedge}$ \\
\hline $\mathrm{FBG}(\mathrm{mM})$ & $5.5 \pm 1.1$ & $5.5 \pm 1.2$ & $5.8 \pm 1.2^{* \wedge}$ & $6.3 \pm 1.3^{* \wedge \#}$ \\
\hline $\mathrm{TG}(\mathrm{mM})$ & $1.6(1.3-2.2)$ & $1.7(1.3-2.2)$ & $1.7(1.3-2.3)$ & $1.8(1.3-2.3)^{* \wedge \#}$ \\
\hline $\mathrm{TC}(\mathrm{mM})$ & $5.0 \pm 0.7$ & $5.0 \pm 0.8$ & $5.1 \pm 0.8$ & $5.1 \pm 0.7$ \\
\hline HDL-c (mM) & $1.2 \pm 0.2$ & $1.2 \pm 0.2$ & $1.0 \pm 0.2^{* \wedge}$ & $1.0 \pm 0.3^{* \wedge} \#$ \\
\hline LDL-c (mM) & $3.0 \pm 0.5$ & $3.1 \pm 0.3^{*}$ & $3.1 \pm 0.4 *$ & $3.2 \pm 0.4^{*}$ \\
\hline eGFR $\left(\mathrm{mL} / \mathrm{min} / 1.73 \mathrm{~m}^{2}\right)$ & $88.1 \pm 13.9$ & $88.3 \pm 16.0$ & $86.3 \pm 15.5$ & $83.2 \pm 12.1^{*}$ \\
\hline hs-TnT (pg/mL) & $3.6(3.0-6.5)$ & $4.6(3.0-7.9)$ & $6.3(4.3-10.1)^{* \wedge}$ & $8.7(5.5-14.2)^{* \wedge \#}$ \\
\hline $\operatorname{IVSTd}(\mathrm{mm})$ & $10.2 \pm 1.4$ & $11.5 \pm 2.3^{*}$ & $12.1 \pm 0.9^{* \wedge}$ & $12.5 \pm 1.5^{* \wedge \#}$ \\
\hline $\operatorname{LVMI}\left(\mathrm{g} / \mathrm{m}^{2}\right)$ & $93.0 \pm 22.6$ & $108.2 \pm 15.9^{*}$ & $169.1 \pm 45.0^{* \wedge}$ & $188.4 \pm 69.8^{* \wedge \#}$ \\
\hline $\operatorname{LVEF}(\%)$ & $63.3 \pm 7.5$ & $62.7 \pm 9.3$ & $65.7 \pm 10.5^{* \wedge}$ & $52.3 \pm 7.8^{* \wedge \#}$ \\
\hline mFS (\%) & $24.1 \pm 4.3$ & $22.9 \pm 5.7^{*}$ & $20.8 \pm 3.1^{* \wedge}$ & $18.6 \pm 6.2^{* \wedge \#}$ \\
\hline E/E'ratio & $6.6 \pm 3.3$ & $8.4 \pm 3.5^{*}$ & $12.7 \pm 2.7^{* \wedge}$ & $14.5 \pm 4.9^{* \wedge} \#$ \\
\hline
\end{tabular}

The normal distributed continuous variables are reported as means $\pm \mathrm{SD}$, and the nonparametrically distributed is reported as median (interquartile range). Categorical variables were presented as frequencies (percentages). Abbreviation are same as Table 1 and Table $2 .{ }^{*} \mathrm{P}<0.05 v s$ normal geometry. ${ }^{\wedge} \mathrm{P}<0.05$ vs concentric remodeling. \#P $<0.05$ vs concentric hypertrophy.

Table 4. Distribution of hs-TnT in subjects with different cardiac remodeling.

\begin{tabular}{|c|c|c|c|c|}
\hline & \multicolumn{2}{|c|}{ Detectable hs-TnT } & \multicolumn{2}{|c|}{ Elevated hs-TnT } \\
\hline & Odds ratio $(95 \% \mathrm{CI})$ & $\mathrm{P}$ & Odds ratio $(95 \% \mathrm{CI})$ & $\mathrm{P}$ \\
\hline HP vs NT & $2.7(1.80-3.60)$ & $<0.001$ & $3.21(3.08-4.02)$ & $<0.001$ \\
\hline HP-CR $v s$ HP-NG & $1.07(0.8-2.45)$ & 0.121 & $1.16(0.79-1.48)$ & 0.157 \\
\hline HP-CH $v s$ HP-NG & $4.18(3.05-9.24)$ & $<0.001$ & $2.91(2.41-6.55)$ & $<0.001$ \\
\hline HP-EH vs HP-NG & $5.34(4.83-10.3)$ & $<0.001$ & $3.25(2.32-7.60)$ & $<0.001$ \\
\hline HP-CH vs HP-CR & $2.15(1.28-3.30)$ & $<0.001$ & $1.93(1.74-3.06)$ & $<0.001$ \\
\hline HP-EH $v s$ HP-CR & $3.02(2.18-5.13)$ & $<0.001$ & $2.64(1.55-3.69)$ & $<0.001$ \\
\hline HP-EH $v s$ HP-CH & $0.89(0.36-1.59)$ & 0.454 & $1.66(1.13-2.76)$ & 0.027 \\
\hline
\end{tabular}

Data are reported as odds ratios and corresponding 95\%CIs. HP = hypertension subjects; NT = normotensive subjects; $\mathrm{NG}=$ Normal geometry; $\mathrm{CR}=$ concentric remodeling; $\mathrm{CH}=$ concentric hypertrophy; $\mathrm{EH}=$ eccentric hypertrophy. Model adjusted for age, gender, current smoking, body mass index, systolic blood pressure, diastolic blood pressure, fasting blood glucose, triglycerides, total plasma cholesterol, high-density lipoprotein cholesterol, low-density lipoprotein cholesterol, estimated glomerular filtration rate, and usage of antihypertensive drugs.

\section{Univariate and multivariate predictors of log-transformed hs-TnT levels}

Univariate and multivariate regression analysis and stepwise multivariable linear regression analyses were performed to evaluated the association between relevant factors and hs-TnT as a continuous variable (natural logarithm transformed). In the univariate linear regression analysis, hs-TnT was related to age, male, smoking, hypertension, diabetes, systolic 
blood pressure, fasting blood glucose, HDL-C, eGFR, LV mass index, mFS and E/E'ratio. In multiple regression analysis, male, hypertension, fasting blood gluscose, eGFR, E/E'ratio and LV mass index were independently associated with hs-TnT levels after adjustment of relevant factors (Table 5).

Table 5. Univariate and multivariate predictors of log-transformed hs-TnT levels.

\begin{tabular}{|c|c|c|c|c|}
\hline \multirow[t]{2}{*}{ Variable } & \multicolumn{2}{|c|}{ Univarite } & \multicolumn{2}{|c|}{ Multivarite } \\
\hline & Standardized $\beta$ & $P$ value & Standardized $\beta$ & $P$ value \\
\hline Age (years) & 0.188 & $<0.001$ & 0.170 & $<0.001$ \\
\hline Male & 0.197 & $<0.001$ & 0.181 & $<0.001$ \\
\hline BMI $\left(\mathrm{kg} / \mathrm{m}^{2}\right)$ & 0.068 & 0.171 & - & - \\
\hline Smoking & 0.083 & 0.009 & - & - \\
\hline Hypertension & 0.160 & $<0.001$ & 0.143 & $<0.001$ \\
\hline Diebetes mellitus & 0.115 & $<0.001$ & - & - \\
\hline $\mathrm{SBP}(\mathrm{mmHg})$ & 0.091 & 0.004 & - & - \\
\hline $\mathrm{DBP}(\mathrm{mmHg})$ & 0.070 & 0.169 & - & - \\
\hline $\mathrm{FBG}(\mathrm{mM})$ & 0.116 & $<0.001$ & 0.101 & $<0.001$ \\
\hline $\mathrm{TG}(\mathrm{mM})$ & 0.013 & 0.752 & - & - \\
\hline HDL-c (mM) & -0.070 & 0.009 & - & - \\
\hline LDL-c (mM) & 0.014 & 0.574 & - & - \\
\hline eGFR & -0.113 & $<0.001$ & -0.084 & 0.012 \\
\hline LVMI & 0.166 & $<0.001$ & 0.127 & $<0.001$ \\
\hline LVEF & -0.020 & 0.190 & - & - \\
\hline $\mathrm{mFS}$ & -0.103 & $<0.001$ & - & - \\
\hline E/E'ratio & 0.124 & $<0.001$ & 0.115 & $<0.001$ \\
\hline
\end{tabular}

High-sensitivity cardiac troponin T levles were natural logarithm transformed. Abbreviations are listed in Table 1 and Table 3.

\section{DISCUSSION}

The results of this study showed that the levels of hs-TnT were higher in hypertensive subjects than in normotensive subjects. The prevalence of detectable and elevated hs-TnT in the normotensive group, the normal geometry group, the concentric remodeling group and the LV hypertrophy groups were gradually increased. Age, gender, hypertension, fasting blood glucose, LVMI, E/E' ratio, and eGFR were independently associated with hs-TnT levels on multiple regression analysis.

Many studies have shown that the left ventricle consistently responds to systemic hypertension by developing concentric and eccentric hypertrophy. A wider spectrum of LV geometric patterns has been demonstrated in hypertensive patients (Georgiopoulou et al., 2010). Studies with hypertensive patients grouped by different geometric patterns have revealed distinctive profiles of hemodynamics, myocardial performance and prognosis (Brown and Haydock, 2000). It has been shown that LV hypertensive remodeling is an important and independent risk factor for cardiovascular events (Rosei and Muiesan, 2004). Hypertensive cardiac remodeling has been characterized by hypertrophy, small vessel coronary artery disease, myocyte disarray, and increased interstitial fibrosis (Frohlich, 2001; Rosei and Muiesan, 2004). However, the usual cardiac troponin assays could not detect the tiny difference in myocyte damage (Martos et al., 2007; Koerbin et al., 2010).

Recent advances have allowed the development of highly sensitive troponin assays, which have greater sensitivity than previous assays and improved precision at 
the lower limits of detection. Several studies have shown the diagnostic and prognostic values of hs-TnT assays across a spectrum of cardiovascular disease, including acute coronary syndromes (Reichlin et al., 2009), stable coronary heart disease (Omland et al., 2009), and even metabolic syndromes and hypertrophic cardiomyopathy (Sato et al., 2003; Moreno et al., 2010). That is, elevated levels of hs-TnT not only provide good sensitivity and specificity for patients with coronary heart disease but also can identify tiny myocardial injury irrespective of the primary diseases. However, the utilization of hs-TnT to estimate cardiac remodeling and function in hypertensive subjects has been limited at present (González et al., 2009).

LV hypertrophy represents the adaptation of the left ventricle to the increase in myocardial strain due to pressure or volume overload and it also becomes an important risk marker of cardiovascular events (Rosei and Muiesan, 2004). In fact, not only the quantity of left ventricular mass, but also its quality (i.e., collagen content, contractile machinery) should be evaluated in LV hypertrophy. hs-TnT could indirectly reflect the quality of LV hypertrophy. These elevated levels of hs-TnT could be due to ongoing myocardial damage or leakage of myofibrillar components and may reflect the continuous loss of viable cardiac myocytes (Pons-Lladó et al., 2000) in the development of hypertensive cardiac remodeling. Besides increased myocardial strain and neuroendocrine system abnormality, cardiomyocyte apoptosis was another important reason for cardiac troponin elevation (Tamura et al., 2000). The balance between cell death and cell survival is a tightly controlled process, especially in terminally differentiated cells, such as cardiomyocyte. Accumulating data support a role for cardiomyocyte apoptosis in the development of hypertensive cardiac remodeling (Fortuno et al., 2003), including the transition from hypertensive compensatory hypertrophy to heart failure. Several molecular and cellular aspects contribute to cardiac injury, involving the inflammatory, myocyte disarray and fibrosis processes (Gai et al., 2010).

LV hypertrophy is often associated with abnormalities of systolic and diastolic function, and its presence clearly predisposes not only to relative cardiac ischemia but also to heart failure (Akintunde et al., 2010). In patients with LV hypertrophy, the decrease in LVM is associated with improved systolic performance at the mid wall and the improvement of diastolic filling (Rosei and Muiesan, 2004). Our study showed a significant association between E/E' ratio and increased hs-TnT levels. LV filling pressures can be estimated with reasonable accuracy by measuring E velocity/flow propagation velocity (E/E'), which is consistent with LV filling pressures (Kouzu et al., 2011). The abnormality of LV filling pressures occurs in early stages of the hypertensive cardiac remodeling process (Cho et al., 2009). LV filling pressure would rise in the presence of increased myocardial strain due to pressure or volume overload even without obvious LV hypertrophy. The process may be involved in tiny cardiac injury independent of greater LV hypertrophy (Tsotetsi et al., 2001).

\section{Clinical meanings}

Circulating hs-TnT levels showed an association with hypertensive cardiac remodeling and diastolic dysfunction. The intricate mechanisms responsible for myocardium remodeling facilitated the development of cardiac dysfunction. In those with LV hypertrophy, a biomarker approach was required for short-term and long-term stratification as well as prog- 
nostication of patients. Biochemical markers may also help to identify patients with no clinical evidence of hypertensive heart disease, and provide information about the need for more aggressive therapy during different stages of the disease.

\section{Limitation}

The principal limitation of the present study lied in its purely cross-sectional design. Long-term follow-up would likely provide additional information to corroborate the proposed value of hs-TnT for predicting progression to clinical states.

\section{Conclusion}

Serum hs-TnT level as an indicator of subclinical ongoing myocyte damage could be a good novel marker to predict and/or indicate deteriorating cardiac remodeling and LV diastolic dysfunction in hypertension.

\section{Conflicts of interest}

The authors declare no potential conflict of interest.

\section{ACKNOWLEDGMENTS}

Research supported by the National Natural Science Foundation of China (\#81270941) and the National Key Basic Research Project of China (\#973), \#2012CB517503, and \#2013CB530804. The authors thank Aloka Corp., Japan for free loan of the $\alpha-10$ apparatus.

\section{REFERENCES}

Akintunde AA, Familoni OB, Akinwusi PO and Opadijo OG (2010). Relationship between left ventricular geometric pattern and systolic and diastolic function in treated Nigerian hypertensives. Cardiovasc. J. Afr. 21: 21-25.

Antman EM (2002). Decision making with cardiac troponin tests. N. Engl. J. Med. 346: 2079-2082.

Apple FS, Jesse RL, Newby LK, Wu AH, et al. (2007). National Academy of Clinical Biochemistry and IFCC Committee for Standardization of Markers of Cardiac Damage Laboratory Medicine Practice Guidelines: Analytical issues for biochemical markers of acute coronary syndromes. Circulation 115: e352-e355.

Arques S, Roux E and Luccioni R (2007). Current clinical applications of spectral tissue Doppler echocardiography (E/E' ratio) as a noninvasive surrogate for left ventricular diastolic pressures in the diagnosis of heart failure with preserved left ventricular systolic function. Cardiovasc. Ultrasound 5: 16.

Brown MJ and Haydock S (2000). Pathoaetiology, epidemiology and diagnosis of hypertension. Drugs 59 Suppl. 2: 1-12.

Cho IJ, Pyun WB and Shin GJ (2009). The influence of the left ventricular geometry on the left atrial size and left ventricular filling pressure in hypertensive patients, as assessed by echocardiography. Korean Circ. J. 39: 145-150.

de Simone G and Devereux RB (2002). Rationale of echocardiographic assessment of left ventricular wall stress and midwall mechanics in hypertensive heart disease. Eur. J. Echocardiogr. 3: 192-198.

Devereux RB, Alonso DR, Lutas EM, Gottlieb GJ, et al. (1986). Echocardiographic assessment of left ventricular hypertrophy: comparison to necropsy findings. Am. J. Cardiol. 57: 450-458.

Fortuno MA, Lopez N, Gonzalez A and Diez J (2003). Involvement of cardiomyocyte survival-apoptosis balance in hypertensive cardiac remodeling. Expert. Rev. Cardiovasc. Ther. 1: 293-307.

Frohlich ED (2001). Fibrosis and ischemia: the real risks in hypertensive heart disease. Am. J. Hypertens. 14: 194S-199S.

Gai X, Zhang Z, Liang Y, Chen Z, et al. (2010). MMP-2 and TIMP-2 gene polymorphisms and susceptibility to atrial fibrillation in Chinese Han patients with hypertensive heart disease. Clin. Chim. Acta 411: 719-724.

Ganau A, Devereux RB, Roman MJ, de SG, et al. (1992). Patterns of left ventricular hypertrophy and geometric remodeling in essential hypertension. J. Am. Coll. Cardiol. 19: 1550-1558. 
Georgiopoulou VV, Kalogeropoulos AP, Raggi P and Butler J (2010). Prevention, diagnosis, and treatment of hypertensive heart disease. Cardiol. Clin. 28: 675-691.

González A, López B, Ravassa S, Beaumont J, et al. (2009). Biochemical markers of myocardial remodelling in hypertensive heart disease. Cardiovasc. Res. 81: 509-518.

Koerbin G, Tate JR and Hickman PE (2010). Analytical characteristics of the Roche highly sensitive troponin T assay and its application to a cardio-healthy population. Ann. Clin. Biochem. 47: 524-528.

Koh YS, Jung HO, Park MW, Baek JY, et al. (2009). Comparison of Left Ventricular Hypertrophy, Fibrosis and Dysfunction According to Various Disease Mechanisms such as Hypertension, Diabetes Mellitus and Chronic Renal Failure. J. Cardiovasc. Ultrasound 17: 127-134.

Kouzu H, Yuda S, Muranaka A, Doi T, et al. (2011). Left ventricular hypertrophy causes different changes in longitudinal, radial, and circumferential mechanics in patients with hypertension: a two-dimensional speckle tracking study. J. Am. Soc. Echocardiogr. 24: 192-199.

Martos R, Baugh J, Ledwidge M, O’Loughlin C, et al. (2007). Diastolic heart failure: evidence of increased myocardial collagen turnover linked to diastolic dysfunction. Circulation 115: 888-895.

McGill D, Talaulikar G, Potter JM, Koerbin G, et al. (2010). Over time, high-sensitivity TnT replaces NT-proBNP as the most powerful predictor of death in patients with dialysis-dependent chronic renal failure. Clin. Chim. Acta 411: 936-939.

Moreno V, Hernandez-Romero D, Vilchez JA, Garcia-Honrubia A, et al. (2010). Serum levels of high-sensitivity troponin T: a novel marker for cardiac remodeling in hypertrophic cardiomyopathy. J. Card. Fail. 16: 950-956.

Omland T, de Lemos JA, Sabatine MS, Christophi CA, et al. (2009). A sensitive cardiac troponin T assay in stable coronary artery disease. N. Engl. J. Med. 361: 2538-2547.

Pons-Lladó G, Ballester M, Borras X, Carreras F, et al. (2000). Myocardial cell damage in human hypertension. J. Am. Coll. Cardiol. 36: 2198-2203.

Reichlin T, Hochholzer W, Bassetti S, Steuer S, et al. (2009). Early diagnosis of myocardial infarction with sensitive cardiac troponin assays. N. Engl. J. Med. 361: 858-867.

Rosei EA and Muiesan ML (2004). Early target organ damage and its reversibility: the heart. Clin. Exp. Hypertens. 26: 673-687.

Sahn DJ, DeMaria A, Kisslo J and Weyman A (1978). Recommendations regarding quantitation in M-mode echocardiography: results of a survey of echocardiographic measurements. Circulation 58: 1072-1083.

Sato Y, Taniguchi R, Nagai K, Makiyama T, et al. (2003). Measurements of cardiac troponin T in patients with hypertrophic cardiomyopathy. Heart 89: 659-660.

Siervo M, Ruggiero D, Sorice R, Nutile T, et al. (2010). Angiogenesis and biomarkers of cardiovascular risk in adults with metabolic syndrome. J. Intern. Med. 268: 338-347.

Tamura T, Said S, Lu W, Harris J, et al. (2000). Is apoptosis present in progression to chronic hypertensive heart failure? J. Card. Fail. 6: 37-42.

Thygesen K, Alpert JS and White HD (2007). Universal definition of myocardial infarction. J. Am. Coll. Cardiol. 50: 2173-2195.

Tsotetsi OJ, Woodiwiss AJ, Netjhardt M, Qubu D, et al. (2001). Attenuation of cardiac failure, dilatation, damage, and detrimental interstitial remodeling without regression of hypertrophy in hypertensive rats. Hypertension 38: 846-851.

Wallace TW, Abdullah SM, Drazner MH, Das SR, et al. (2006). Prevalence and determinants of troponin T elevation in the general population. Circulation 113: 1958-1965.

Xu R, Ye P, Luo L, Xiao W, et al. (2011). Association between high-sensitivity cardiac troponin T and predicted cardiovascular risks in a community-based population. Int. J. Cardiol. 149: 253-256. 